I. M. Aberbach

Nagoya Math. J.

Vol. 135 (1994), 43-54

\title{
TIGHT CLOSURE IN F-RATIONAL RINGS
}

\author{
IAN M. ABERBACH
}

\section{Introduction}

All rings are commutative with unit and modules are unital. With one exception all rings are Noetherian. We consider only rings of positive characteristic $p$. Section 0 contains background, definitions and terminology.

Peskine and Szpiro showed in [PS] that when the ring $R$ has positive characteristic the Frobenius endomorphism may be exploited with strong results. More recently, Hochster and Huneke have developed the theory of tight closure and obtained numerous results for equicharacteristic rings containing a field. If $R$ is a Noetherian ring of characteristic $p$ and $I$ is an ideal of $R$, then $x \in R$ is in the tight closure of $I$, denoted $I^{*}$, if there exists an element $c$ not in any minimal prime of $R$ such that $c x^{p^{e}} \in I^{\left[p^{e}\right]}=\left(i^{p^{e}}: i \in I\right) R$ for all $e \gg 0$. Using the Frobenius endomorphism there is a definition of tight closure for finitely generated modules as well. When a module $N \subseteq M$ is equal to its tight closure then we say that $N$ is tightly closed in $M$. See $\S 0$ for a more thorough exposition of tight closure. Statements which may be proved in characteristic $p$ using tight closure arguments may then often be proved in the equicharacteristic 0 case using the techniques of Artin approximation (see $[\mathrm{H}]$ ).

The impetus for this paper was the question: if $I \subseteq R$ is tightly closed and $R / I$ has finite projective dimension (or more generally, finite phantom projective dimension) then is $I^{\left[p^{e}\right]}$ tightly closed and what other modules of finite (phantom) projective dimension have their zero submodule tightly closed? The key to the answers we have obtained are contained in Lemmas 1.2 and 1.3 , in which we note that, given a chain map of finite free acyclic complexes, certain tight closure modules determined by the complexes are related.

When $(R, m)$ is local and every ideal generated by parameters is tightly closed then $R$ is called $\mathrm{F}$-rational (see [HH3, §4]). When $R$ is equidimensional and the homomorphic image of a Cohen-Macaulay ring then it suffices that one full system of parameters be tightly closed, in which case $R$ is Cohen-Macaulay. In

Received April 27, 1992. 
particular, if $\mathbf{x}=x_{1}, \ldots, x_{d}$ is a system of parameters for $R$ then the Koszul complex $K .\left(x_{1}, \ldots, x_{d} ; R\right)$ is a free resolution of $R /(\mathbf{x})$. Our first result, Theorem 1.1, shows that when the local ring $R$ is Cohen-Macaulay then $R$ is $\mathrm{F}$-rational if and only if there exists some $N \subseteq M$ with $N_{M}^{*}=N$ and $\operatorname{pd} M / N=\operatorname{dim} R$, in which case $N_{M}^{*}=N$ for all $N \subseteq M$ with pd $M / N<\infty$. It is natural to ask whether the existence of a tightly closed module $N \subseteq M$ such that $M / N$ has finite phantom projective dimension equal to $\operatorname{dim} R$ implies that $R$ is Cohen-Macaulay. Corollary 1.5 shows that with mild conditions on $(R, m), R$ is $\mathrm{F}$-rational when such a pair exists and $M / N$ is isomorphic to $R / J$ where $J$ is an $m$-primary monomial ideal in parameters. This result generalizes [HH3, Theorem $4.2 \mathrm{~d}$ ].

In Section 2 we turn to the case of a finitely generated module $M$ of finite projective dimension in which 0 is tightly closed. Theorem 2.1 shows that in this case, under mild conditions on $R, 0$ is tightly closed in $\mathbf{F}^{e}(M)$ for all $e \geq 0$ (see $\S 0$ for a definition of $\mathbf{F}^{e}$ ). Additionally, when $R$ is local and $\operatorname{pd} M<\operatorname{dim} R$ we show that the depth of $R$ must be greater than the projective dimension of $M$. This result generalizes [HH3, Theorem $4.3 \mathrm{~b}$ ].

The main result of [HH2], Theorem 5.15 , states that if $(R, m)$ is a bi-equidimensional excellent semi-local domain of characteristic $p, K$ is the fraction field of $R$, and $L$ is an algebraic closure of $K$ then the integral closure of $R$ in $L$, denoted $R^{+}$, is a balanced big Cohen-Macaulay algebra for $R$ (by balanced we mean that every system of parameters for $R$ is a regular sequence on $R^{+}$). The ring $R^{+}$is not Noetherian if $\operatorname{dim} R>0$.

Let $I \subseteq R$ be an ideal. By the plus closure of $I$ we mean $I^{+}=I R^{+} \cap R$. Note that if $x \in I^{+}$then there is a module finite extension domain $S \supseteq R$ such that $x \in I S$. For finitely generated $R$-modules $N \subseteq M$, the plus closure of $N$ in $M$ is $N^{+}=\operatorname{ker}\left(M \rightarrow R^{+} \bigotimes_{R} M / N\right)$. It is easy to see that $N_{M}^{+} \subseteq N_{M}^{*}$. Recent work of K. Smith [S] shows that if $I$ is generated by parameters then $I^{*}=I^{+}$. We extend ths result in Section 3 by showing that knowing tight closure equals plus closure for parameter ideals forces plus closure to equal tight closure for $N \subseteq M$ when $M / N$ has finite phantom projective dimension, a much wider class of module pairs.

I wish to thank Melvin Hochster and Craig Huneke for many helpful conversations concerning this material. 


\section{Background}

In this section we discuss the key definitions and notational conventions that we will use.

Definition. The $\operatorname{ring} R$ is equidimensional if $\operatorname{dim} R=\operatorname{dim} R / P$ for every minimal prime $P$ of $R$.

Definition. Let $R$ be a Noetherian ring and let $I \subseteq R$ be an ideal. Then the minheight of $I$ is minht $I=\min \{h t I(R / P): P$ is a minimal prime of $R\}$. When $R$ is catenary and equidimensional then minht $I=$ ht $I$.

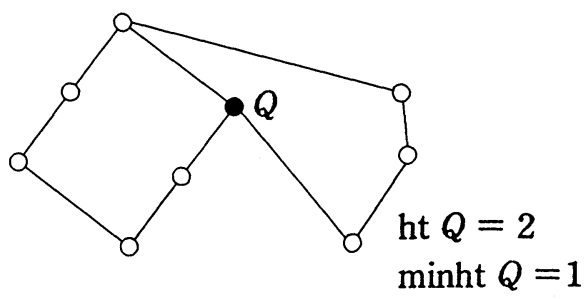

Tight closure. (For more information see $[\mathrm{HH} 1, \S \S 4,8]$.) $\quad$ Let $R$ be a Noetherian ring of positive prime characteristic $p$. Let $R^{\circ}=R-\cup$ \{minimal primes of $R\}$. We will use $e$ to denote a non-negative integer and $q=p^{e}$ the corresponding power of $p$. Then $I^{[q]}=\left(i^{q}: i \in I\right)$ is the $q$ th Frobenius power of $I$. We say that an element $x \in R$ is in the tight closure of $I$, denoted $I^{*}$, if there exists $c \in R^{\circ}$ such that $c x^{q} \in I^{[q]}$ for all $q \gg 0$.

The Frobenius endomorphism $f^{e}: R \rightarrow R$ is given by $f^{e}(r)=r^{p^{e}}$. If we let $S_{e}$ denote $R$ as an $R$-algebra under $f^{e}$ then the functor $\mathbf{F}^{e}$ is $S_{e} \otimes_{R}$. Since $S_{e}=R$, $\mathbf{F}^{e}$ is a covariant functor from $R$-modules to $R$-modules.

Given a module $M$ and an element $m \in M$ then by $m^{q}$ we mean $1 \otimes m \in$ $\mathbf{F}^{e}(M)$. If $N \subseteq M$ then $N^{[q]}$ is the submodule of $\mathbf{F}^{e}(M)$ generated by $\left\{n^{q}: n \in N\right\}$. An element $m \in M$ is in the tight closure of $N$ in $M$, denoted $N_{M}^{*}$, if there exists $c$ $\in R^{\circ}$ such that $\mathrm{cm}^{q} \in N^{[q]}$ for all $q \gg 0$. If $N_{M}^{*}=N$ we say that $N$ is tightly closed in $M$.

Note that by the right exactness of tensor, $m \in M$ is in the tight closure of $N$ if and only if $m+N$ is in the tight closure of 0 in $M / N$. We will use this observation in the following way: if $M=\operatorname{cok}\left(G_{1} \stackrel{a}{\rightarrow} G_{0}\right)$ then $0_{M}^{*}$ corresponds to $(\operatorname{im} a)_{G_{0}}^{*}$. 
Definition. A local ring is $F$-rational if every ideal generated by part of a system of parameters is tightly closed (see [HH3, §4] and [FW]).

Theorem 1.1 makes it clear that local F-rational rings have considerably stronger tight closure properties.

Definition. Let $c \in R^{\circ}$ and let $q^{\prime}$ be a positive integer. If for all finitely generated modules $N \subseteq M$ and for all $x \in N_{M}^{*}, c x^{q} \in N^{[q]}$ for all $q \geq q^{\prime}$ then $c$ is called a $q^{\prime}$-weak test element.

Maps of Free Modules. Let $L$ and $G$ be free $R$-modules of rank $s$ and $t$ respectively. Let $\phi: L \rightarrow G$ be a homomorphism. If we choose bases for $L$ and $G$ then the map $\phi$ is given by a $t \times s$ matrix $A=\left[a_{i j}\right]$ with entries in $R$. By $I_{i}(\phi)$ we mean the ideal of $R$ generated by all the $i \times i$ minors of $A\left(I_{i}(\varphi)\right.$ is independent of the chosen bases). We denote $\mathbf{F}^{e}(\phi)$ by $\phi^{[q]}$. The map $\phi^{[q]}$ is given by the matrix $A^{[q]}=\left[a_{i j}^{q}\right]$. Thus if $M=\operatorname{cok} \phi$ then $\mathbf{F}^{e}(M)=\operatorname{cok} \phi^{[q]}$.

Given an $R$-module $M$, let $M^{\vee}=\operatorname{Hom}_{R}(M, R)$ be its dual. If $L, G$, and $\phi$ are as above then $\phi^{\vee}: G^{\vee} \rightarrow L^{\vee}$ and $\phi^{\vee}$ is given by the $s \times t$ matrix $A^{T}=$ $\left[a_{i j}\right]$ with respect to the dual bases. Thus it is clear that $\left(\phi^{\vee}\right)^{[q]}=\mathbf{F}^{e}\left(\phi^{\vee}\right)$ is the same as $\left(\phi^{[q]}\right)^{\vee}$. We will freely make this identification, as well as identifying $\phi^{\vee \vee}$, $G^{\vee \vee}$, and $L^{\vee \vee}$ with $\phi, G$, and $L$ respectively.

Parameters and iterated operations. Let $R$ be a Noetherian ring and let $\mathbf{x}=$ $x_{1}, \ldots, x_{n}$ be a sequence of elements in $R$. The ideal $I=(\mathbf{x})$ is said to be a parameter ideal if ht $I R_{P} \geq n$ for all primes $P$ containing $I$. We say that the ideal $J \subseteq R$ is a monomial ideal in parameters if $J=\left(m_{1}, \ldots, m_{t}\right) R$ where each $m_{i}$ is a monomial in $\mathbf{x}$.

When $\mathbf{x}=x_{1}, \ldots, x_{d}$ is a suitable $R$-sequence (see [HH1, §7.3]) and $J_{1}, J_{2}$ are two monomial ideals in $\mathbf{x}$ then $\left(J_{1}: J_{2}\right)$ may be computed formally and is also a monomial ideal in $\mathbf{x}$. The results of [HH1, §7], [HH4] and [AHH] show that when $\mathbf{x}$ consists of parameters (and all subsets have suitable minheight) then $\left(J_{1}: J_{2}\right)$ is contained in the tight closure of the formal answer - in this situation we say that "tight closure captures the colon." A much more general theory of "iterated operations," involving sum, product and intersection (as well as colon), is developed in [HH1] (see, in particular, Theorem 7.15).

We note here that iterated operations applies in the following cases:

(1) If $R$ is a locally equidimensional image of a Cohen-Macaulay ring and $x_{1}$, $\ldots, x_{d}$ are permutable parameters in $R$ then iterated operations applies 
to the set of monomial ideals in $x_{1}, \ldots, x_{d}$.

(2) If $R$ is a locally equidimensional module-finite extension of a regular domain $A$ then iterated operations applies to the set of $R$-ideals extended from $A$.

Phantom projective dimension and acceptable type. Let $G$. be a left complex of finitely generated projective modules. We say that $G$. is stably phantom acyclic if for all $e \geq 0$ and for all $i \geq 1$, the $i$ th cycles of $\mathbf{F}^{e}(G$.$) are contained in the tight$ closure of the $i$ th boundaries (in $\mathbf{F}^{e}\left(G_{i}\right)$ ). The complex $G$. is then called a phantom resolution of $M=H_{0}(G$.$) . [A, Theorem 2.1.7] shows that if M$ has a phantom resolution then it has one that is finite. We write $\operatorname{ppd}_{R} M<\infty$. Phantom homology and phantom projective dimension are studied in [HH1], [HH4], [A] and [AHH]. Finite phantom projective dimension is a generalization of finite projective dimension. Indeed, the phantom acyclicity criterion (given in various forms in [HH1] and [HH4] but stated in a stronger form as [AHH, Theorem 5.3]) is a generalization of the Buchsbaum-Eisenbud criterion for acyclicity [BE, Corollary 1]. The phantom acyclicity criterion gives necessary and sufficient conditions for a complex to be stably phantom acyclic (with mild conditions on the ring $R$ ). Rings for which the sufficiency conditions imply stably phantom homology are said to satisfy the phantom acyclicity criterion. If, in addition, $R$ is universally catenary and for all primes $P \in \operatorname{Spec} R$ and all indeterminates $T_{1}, \ldots, T_{n}$ the ring $\left(R\left[T_{1}, \ldots\right.\right.$, $\left.\left.T_{n}\right]\right)_{P R\left[T_{1}, \ldots, T_{n}\right]}$ satisfies the phantom acyclicity criterion, we say that $R$ is of acceptable type.

\section{Ideals of finite projective dimension are tightly closed in F-rational rings}

The main goal of this section is to prove:

THEOREM 1.1. Let $(R, m)$ be a local Cohen-Macaulay ring of dimension d. Then the following are equivalent:

(1) $R$ is F-rational.

(2) There exists a system of parameters $\mathbf{x}=x_{1}, \ldots, x_{d}$ such that $(\mathbf{x})$ is tightly closed in $R$.

(3) For all ideals $I \subseteq R$ such that $R / I<\infty$, the ideal $I$ is tightly closed.

(4) For all finitely generated modules $M$ such that $\mathrm{pd} M<\infty$, the zero submodule is tightly closed in $M$.

(5) There exists a tightly closed ideal I such that $\mathrm{pd} R / I=d$.

(6) There exists a finitely generated module $M$ of projective dimension $d$ such 
that 0 is tightly closed in $M$.

The key observation for proving Theorem 1.1 is the following lemma:

Lemma 1.2. Let $R$ be a Noetherian ring of characteristic $p$. Let $\left(L_{.}, a.\right)$ and $(G,, b$.$) be complexes of finitely generated free modules of length d$ where $L$. is acyclic. If $\phi_{.}: G . \rightarrow L$. is a chain map and $\operatorname{im} \phi_{0} \subseteq\left(\operatorname{im} a_{1}\right)_{L_{0}}^{*}$ then

(1) $\operatorname{im} \phi_{d} \subseteq\left(I_{1}\left(b_{d}\right) L_{d}\right)_{L_{d}}^{*}$, and

(2) $\operatorname{im} \phi_{d}^{\vee} \subseteq\left(\operatorname{im~} b_{d}^{\vee}\right)_{G_{d}^{\vee}}^{*}$.

Proof. Since $\operatorname{im} \phi_{0} \subseteq\left(\operatorname{im} a_{1}\right)_{L_{0}}^{*}$, by [HH1, Theorem 8.5d] there exists $c \in R^{\circ}$ such that $c\left(\operatorname{im} \phi_{0}^{[q]}\right) \subseteq \operatorname{im} a_{1}^{[q]}$ for $q \gg 0$. Thus, for $q \gg 0$ we have the composite diagram:

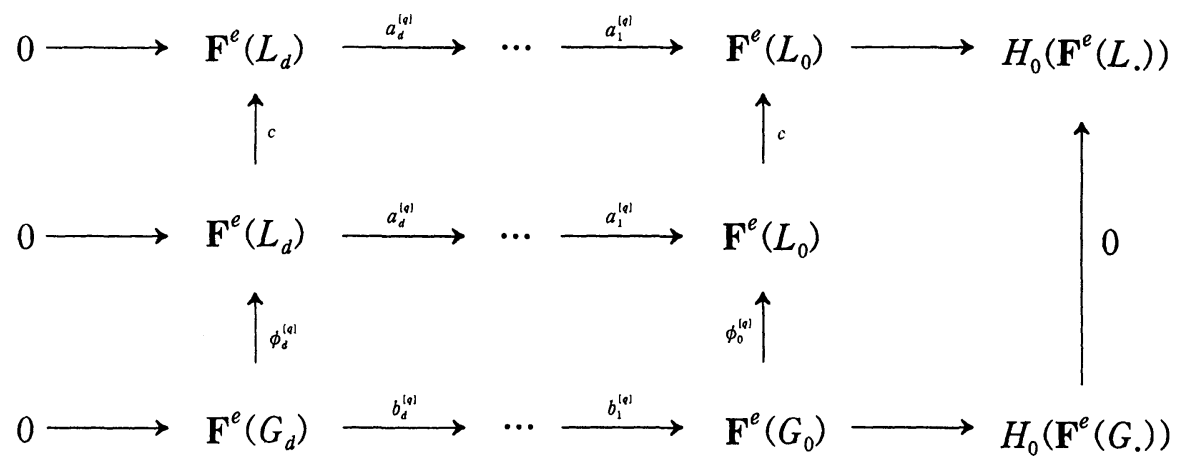

Note that $\mathbf{F}^{e}(L$.$) remains acyclic for all e \geq 0$ [PS, Theorem 1.7]. Thus by [CE, Chap. V, Proposition 1.1] the composite chain map is homotopic to the zero chain map. Hence we get a commutative diagram:

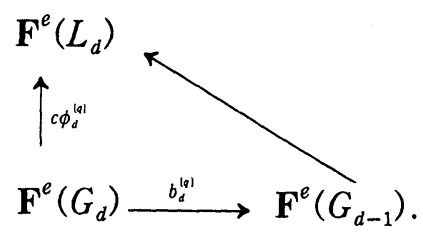

Thus $c\left(\operatorname{im} \phi_{d}^{[q]}\right)=c\left(\operatorname{im} \phi_{d}\right)^{[q]} \subseteq I_{1}\left(b_{d}\right)^{[q]} \mathbf{F}^{e}\left(L_{d}\right)=\left(I_{1}\left(b_{d}\right) L_{d}\right)^{[q]}$. This proves statement (1).

Dualizing (\#) yields 
(*)

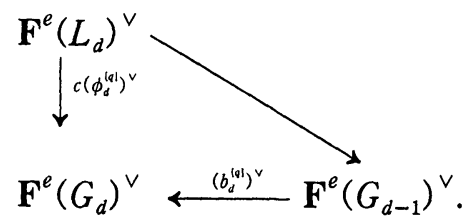

Thus $c\left(\operatorname{im}\left(\phi_{d}^{\vee}\right)^{[q]}\right)=c\left(\operatorname{im} \phi_{d}^{\vee}\right)^{[q]} \subseteq \operatorname{im}\left(\left(b_{d}^{\vee}\right)^{[q]}\right)=\left(\operatorname{im} b_{d}^{\vee}\right)^{[q]}$. Hence im $\phi_{d}^{\vee} \subseteq(\operatorname{im}$ $\left.b_{d}^{\vee}\right)_{G \searrow}^{*}$. This proves statement (2).

We will use Lemma 1.2 in the case where the appropriate image is tightly closed.

Lemma 1.3. Let $R$ be a commutative ring. Let $\left(L_{.}, d.\right)$ and $(G .$, e. $)$ be length $n$ complexes of finitely generated free modules such that $G^{\vee}$. is acyclic. Let $\phi_{.}: G . \rightarrow L$. be a chain map. If $\operatorname{im} \phi_{n}^{\vee} \subseteq \operatorname{im} e_{n}^{\vee}$ then $\operatorname{im} \phi_{o} \subseteq \operatorname{im} d_{1}$.

Proof. The hypotheses give that the dual chain map $\phi^{\vee}$. induces the zero map from $H_{0}\left(L^{\vee}\right)$ to $H_{0}\left(G_{.}^{\vee}\right)$, hence is chain homotopic to the zero map. At the last stage the chain homotopy looks like:

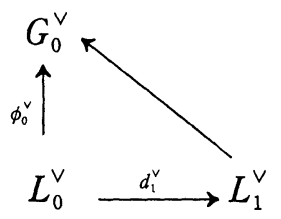

which when dualized yields the desired result.

We are now ready to prove Theorem 1.1 .

Proof (of Theorem 1.1). The equivalence of (1) and (2) is [HH3, Theorem $4.2 \mathrm{~d}$ ]. The implications 4$) \Rightarrow 3) \Rightarrow 1$ ) and 4$) \Rightarrow 5) \Rightarrow 6$ ) are clear because parameter ideals have finite projective dimension in a Cohen-Macaulay ring.

We first prove 1$) \Rightarrow 4$ ). Since $R$ is Cohen-Macaulay and F-rational, [HH3, Theorem 4.2f] shows that this condition localizes. Also, $\left(0_{M}^{*}\right)_{P} \subseteq\left(0_{P}\right)_{M_{p}}^{*}$ for all $P \in \operatorname{Spec} R$. If 0 is not tightly closed choose a nonzero $u \in 0_{M}^{*}$. We may localize at a prime in Ass $R u$, and hence assume that there is a system of parameters $\mathbf{x}=$ $x_{1}, \ldots, x_{d}$ such that $(\mathbf{x}) u=0$. Note that this implies that $\operatorname{pd} M=\operatorname{dim} R$.

Let $\left(G ., a_{0}\right)$ be a minimal resolution of $M$ and let $\phi_{0}: R \rightarrow G_{0}$ be given by 
$\phi_{0}(1)=w$, where $w$ maps to $u$ in $M$. The map $\phi_{0}$ induces $\alpha: R /(\mathbf{x}) \rightarrow M$ where $\alpha(1+(\mathbf{x}))=u$, so $\phi_{0}$ lifts to a chain map $\phi_{.}: K .(\mathbf{x} ; R) \rightarrow G$. (where $K .(\mathbf{x} ; R)$ is the Koszul complex of $x_{1}, \ldots, x_{d}$ on $R$ ). Since im $\phi_{0}=R w \subseteq\left(\operatorname{im} a_{1}\right)_{G_{0}}^{*}$, Lemma 1.2 (1) shows that $\operatorname{im} \phi_{d} \subseteq\left((\mathbf{x}) G_{d}\right)_{G d}^{*}=(\mathbf{x}) G_{d}$ (the last equality is because $R$ is F-rational). We may now apply Lemma 1.3 to conclude that $w \in \operatorname{im} a_{1}$, i.e. that $u=0$, a contradiction. Thus 0 is tightly closed in $M$.

We now prove 6$) \Rightarrow 2$ ). Let $M$ have a minimal finite free resolution $(G ., a$. of length $d$ and suppose that 0 is tightly closed in $M$. Let $\mathbf{x}=x_{1}, \ldots, x_{d}$ be a system of parameters. We need to show that $(\mathbf{x})$ is tightly closed.

If not, choose $w \in(\mathbf{x})^{*}-(\mathbf{x})$ such that $m w \subseteq(\mathbf{x})$ (this is possible since $(\mathbf{x})$ is $m$-primary). We will construct a chain map from $G^{\vee}$ to $K .(\mathbf{x} ; R)$. Fix a basis for the free module $G_{d}$, so that we have a corresponding dual basis for $G_{d}^{\vee}$. Let $\mathbf{e}_{1}$ be a member of the dual basis for $G_{d}^{\vee}$. Let $\phi_{0}: G_{d}^{\vee} \rightarrow R$ be given by extending $\phi_{0}\left(\mathbf{e}_{1}\right)=w$, and $\phi_{0}\left(\mathbf{e}_{1}\right)=0$ for $i>1$. Since the resolution is minimal and $m w \subseteq$ $(\mathbf{x})$, there is an induced map from $H_{0}\left(G^{\vee}\right)$ to $R /(\mathbf{x})$, from which we obtain the desired chain map, $\phi$.

By Lemma $1.2(2), \operatorname{im} \phi_{d}^{\vee} \subseteq\left(\operatorname{im} a_{1}^{\vee \vee}\right)_{G_{0}^{\vee \vee}}^{*}=\operatorname{im} a_{1}$ (the equality comes from identifying the double dual and noting that $\operatorname{im} a_{1}$ is tightly closed in $G_{0}$ ). We may now apply Lemma 1.3 to conclude that $w \in(\mathbf{x})$, a contradiction. Therefore, the ideal (x) is tightly closed.

In Theorem 1.1 we assumed that $R$ was Cohen-Macaulay in order to obtain our results. As [HH3, Theorem 4.3] shows, an equidimensional local homomorphic image of a Cohen-Macaulay ring in which some system of parameters is tightly closed must be Cohen-Macaulay. We extend this result in Corollary 1.5, using the properties of iterated operations of monomial ideals in parameters.

LEMMA 1.4. Let $(R, m)$ be any local ring in which tight closure captures the colon for monomial ideals in parameters. Let $J=\left(m_{1}, \ldots, m_{l}\right)$ be an ideal of monomials in parameters, one of which is $x$. Suppose that $m_{i}=x m_{i}^{\prime}$ for $1 \leq i \leq t$ and $x$ does not divide $m_{i}$ for $i>t$.

If $J$ is tightly closed then so is $J^{\prime}=\left(m_{1}^{\prime}, \ldots, m_{t}^{\prime}, m_{t+1}, \ldots, m_{l}\right)$.

Proof. Let $u \in\left(J^{\prime}\right)^{*}$. Then $x u \in x\left(J^{\prime}\right)^{*} \subseteq J^{*}=J$. Thus

$$
x u=\sum_{i=1}^{l} a_{i} m_{i}=x \sum_{i=1}^{t} a_{i} m_{i}^{\prime}+\sum_{i=t+1}^{l} m_{i}
$$




$$
u-\sum_{i=1}^{t} a_{i} m_{i}^{\prime} \in\left(m_{t+1}, \ldots, m_{l}\right): x \subseteq\left(m_{t+1}, \ldots, m_{l}\right)^{*} \subseteq J
$$

Therefore $u \in J^{\prime}$.

Corollary 1.5. Let $(R, m)$ be any local equidimensional ring in which tight closure captures the colon for monomial ideals in parameters. If $\operatorname{dim} R=d$, the sequence $\mathbf{x}=x_{1}, \ldots, x_{d}$ is a system of parameters and $J=\left(x_{1}^{r_{1}}, \ldots x_{d}^{r_{d}}, m_{1}, \ldots, m_{l}\right)$ is a tightly closed monomial ideal in $\mathbf{x}$ then for some positive integers $b_{1}, \ldots, b_{d}$ the ideal $\left(x_{1}^{b_{1}}, \ldots, x_{d}^{b_{d}}\right)$ is tightly closed.

In particular, $R$ is Cohen-Macaulay and $F$-rational.

Proof. The last statement follows from the first statement and from [HH3, Theorems 4.2, 4.31.

We prove the first statement by induction on $n=\sum_{1}^{l} \operatorname{deg}\left(m_{i}\right)$. If $n=0$ then there are no monomials so there is nothing to prove. Suppose that $n>0$. Then without loss of generality we may assume that for some $t \geq 1, x_{1}$ divides the first $t$ monomials and does not divide the last $l-t$ monomials, say $m_{i}=x_{1} m_{1}^{\prime}$ for $1 \leq i$ $\leq t$. Note that this implies that $r_{1} \geq 2$. Then by Lemma 1.4 we see that $J^{\prime}=$ $\left(x_{1}^{\gamma_{1}-1}, x_{2}^{r_{2}}, \ldots, x_{d}^{\gamma_{d}}, m_{1}^{\prime}, \ldots, m_{t}^{\prime}, m_{t+1}, \ldots, m_{l}\right)$ is tightly closed. The sum of degrees of the mixed monomials (i.e. not a power of one parameter) in a minimal generating set of $J^{\prime}$ will be less than or equal to $n-t$.

Recall that iterated operations may be applied in more general circumstances. We ask the following:

Question 1.6. If $(R, m)$ is equidimensional and module-finite over the regular ring $(A, n)$ and $J R$ is tightly closed for some $n$-primary ideal of $A$ then is $R$ Cohen-Macaulay (hence $\mathrm{F}$-rational)?

In the situation of Question 1.6, $A / J$ has a finite free resolution $G$. over $A$. The complex $R \otimes_{A} G$. is then a finite phantom resolution of $R / J R$ over $R$ (see [A], $[\mathrm{AHH}])$. This observation leads to the more general:

Question 1.7. If $(R, m)$ is equidimensional of dimension $d$ and $R$ has a finitely generated module $M$ such that $\operatorname{ppd}_{R} M=d$ and 0 is tightly closed in $M$ then is $R$ Cohen-Macaulay (hence $\mathrm{F}$-rational)? 


\section{Tightly closed ideals of finite projective dimension}

THEOREM 2.1. Let $R$ be a ring of acceptable type containing a weak test element. Suppose that $M$ is a finitely generated module of finite projective dimension. If 0 is tightly closed in $M$ then 0 is tightly closed in $\mathbf{F}^{e}(M)$ for all $e \geq 0$.

In particular, if $I \subseteq R$ is tightly closed and $\mathrm{pd} R / I<\infty$ then $I^{[q]}$ is tightly closed for all $q$.

Proof. It clearly suffice to prove the claim for $\mathbf{F}^{1}(M)$. Suppose the claim is false. Let $z \in 0_{\mathbf{F}^{1}(M)}^{*}$ be nonzero. We may localize at a prime $P$ in Ass $R z$, since $z / 1$ will then be in the tight closure of 0 in $\mathbf{F}^{1}\left(M_{P}\right)$ and $0_{M_{P}}^{*}=\left(0_{M}^{*}\right)_{P}=0_{P}=0$ by [AHH, Theorem 8.1]. Thus we may assume that $(R, m)$ is local (of acceptable type), $\operatorname{dim} R=d=\operatorname{pd} M$, and by taking a multiple of the original $z$, that $m z=0$.

Let $\left(G ., a\right.$.) be a minimal free resolution of $M$. Then $\mathbf{F}^{1}(G$.$) is a minimal free$ resolution of $\mathbf{F}^{1}(M)$. Let $w \in \mathbf{F}^{1}\left(G_{0}\right)$ be an element representing $z$. As in the proof of Theorem 1.1 $(6 \Rightarrow 2)$, we have a map $\phi_{0}: G_{d}^{\vee} \rightarrow \mathbf{F}^{1}(G$.) which induces a map cok $a_{d}^{\vee} \rightarrow \mathbf{F}^{1}(M)$ since $m z=0$. The map $\phi_{0}$ extends to a chain map $\phi .: G^{\vee}$. $\rightarrow \mathbf{F}^{1}(G$.).

By Lemma $1.2(2), \operatorname{im} \phi_{d}^{\vee} \subseteq\left(\operatorname{im} a_{1}\right)_{G_{0}}^{*}=\operatorname{im} a_{1}$. Thus by Lemma $1.3, w \in \operatorname{im}$ $a_{1}$, i.e. $z=0$, a contradiction. Therefore 0 is tightly closed in $M$.

The next proposition shows that when a module of finite projective dimension with zero tightly closed exists then the local ring $R$ must have a large enough depth.

PROPOSITION 2.2. Let $(R, m)$ be any equidimensional ring in which tight closure captures the colon for monomial ideals in parameters. Suppose that $M$ is a finitely generated $R$-module such that $\mathrm{pd} M=t<\operatorname{dim} R$ and $0_{M}^{*}=0$. Then $\operatorname{depth}_{m} R \geq$ $t+1$.

Proof. By the Auslander-Buchsbaum theorem, $\operatorname{depth}_{m} R \geq t$. Suppose that $\operatorname{depth}_{m} R=t$. Let $\mathbf{x}=x_{1}, \ldots, x_{t}$ be a maximal $R$ sequence in $m$. Then $m \in$ Ass $R /(\mathbf{x})$, so choose $u \in R \backslash(\mathbf{x})$ such that $m u \subseteq(\mathbf{x})$. By iterated operations, if $\mathbf{x}, v$ is part of a system of parameters then $u \in(\mathbf{x}): v \subseteq(\mathbf{x})^{*}$.

Let $\phi_{0}: G_{t}^{\vee} \stackrel{[u 0 \ldots . .0]}{\longrightarrow} R$ be a map as in the previous theorem. The map $\phi_{0}$ extends to a chain map $\phi .: G^{\vee} \rightarrow K .(\mathbf{x} ; R)$ since $m u \subseteq(\mathbf{x})$. Now we conclude exactly as in the last paragraph of the preceding proof that $u \in(\mathbf{x})$, a contradiction. 


\section{Tight closure and plus closure for ideals of finite (phantom) projective dimension}

Recall that if $(R, m)$ is an excellent semi-local domain then $R^{+}$is a balanced big Cohen-Macaulay algebra for $R$, and we are led to the notion of plus closure. $\mathrm{K}$. Smith has shown that if $R$ is locally excellent and $I$ is generated locally by parameters then $I^{*}=I^{+}$([Sm, Theorem 5.1]). It is an open question whether plus closure equals tight closure in general. We have, however, the following result which connects the question for parameters with that for modules of finite phantom projective dimension.

THEOREM 3.1. Let $R$ be a locally excellent domain. Let $N \subseteq M$ be finitely generated modules such that $\operatorname{ppd} M / N \leq \infty$. Then $N^{+}=N^{*}$.

Proof. We need only show that $N^{*} \subseteq N^{+}$. Suppose not and let $z \in N^{*} \backslash N^{+}$. By localizing and replacing $z$ by a multiple we may assume that $m z \subseteq N^{+}$. Let $\left(G, a\right.$ ) be a minimal resolution of $M / N$ of length $n$, let $w \in G_{0}$ represent $z$ and let $\mathbf{x}=x_{1}, \ldots, x_{n}$ be part of a system of parameters for $R$. Since $R^{+} \bigotimes_{R} G$. is acyclic, the map $\phi_{0}: R^{+} \rightarrow R^{+} \bigotimes_{R} G_{0}$ sending 1 to $1 \otimes w$ extends to a chain map $\phi .: K .\left(\mathbf{x} ; R^{+}\right) \rightarrow R^{+} \otimes_{R} G$. This chain map will be the extension of a chain map $\phi^{\prime}$. in some module finite extension domain $S$ of $R$. Thus, im $\phi_{n}^{\prime} \subseteq(\mathbf{x})^{*} S \otimes_{R} G_{n}=$ $(\mathbf{x})^{+} S \otimes_{R} G_{n}$ by Lemma 1.2. Thus we can apply Lemma 1.3 to $\phi . \otimes_{R} R^{+}$to get that $1 \otimes w \in \operatorname{im}\left(1 \otimes a_{1}\right)$ i.e., that $z \in N^{+}$.

The following corollary was pointed out by Brian MacCrimmon. This result along the lines of [AHH, Theorem 8.1] follows from Theorem 3.1 in the excellent case.

Corollary 3.2. Let $R$ be a locally excellent ring. If $\operatorname{ppd} M / N<\infty$ then the tight closure of $N$ in $M$ commutes with localization for all localizations of $R$.

Proof. In every localization, the tight closure and the plus closure of $N$ in $M$ are equal, and plus closure localizes [HH2, Lemma 6.5]

\section{REFERENCES}

[A] I. M. Aberbach, Finite phantom projective dimension, Amer. J. Math., 116 (1994), 447-477.

[AHH] I. M. Aberbach, M. Hochster and C. Huneke, Localization of tight closure and 
modules of finite phantom projective dimension, J. reine angew. Math., 434 (1993), 67-114.

[BE] D. A. Buchsbaum and D. Eisenbud, What Makes a Complex Exact?, J. Algebra, 25 (1973), 259-268.

[CE] H. Cartan and S. Eilenberg, Homological Algebra, Princeton University Press, Princeton, 1956.

[FW] R. Fedder and K. Watanabe, A characterization of F-regularity in terms of F-purity, Commutative Algebra (Proceedings of a Microprogram held June 15-July 2, 1987), Mathematical Sciences Research Institute Publications, vol. 15, Springer-Verlag, New York-Berlin-Heidelberg-London-Paris-Tokyo, 1989, pp. $227-245$.

[H] M. Hochster, Topics in the homological theory of modules over commutative rings, C.B.M.S. Regional Conf. Ser. in Math. no. 24, Amer. Math. Soc., Providence, R. I., 1975.

[HH1] M. Hochster and C. Huneke, Tight closure, invariant theory, and the BriançonSkoda theorem, J. Amer. Soc., 3 (1990), 31-116.

[HH2] M. Hochster and C. Huneke, Infinite integral extensions and big Cohen-Macaulay algebras, Annals of Math., 135 (1992), 53-89.

[HH3] M. Hochster and C. Huneke, F-regularity, test elements, and smooth base change, preprint.

[HH4] M. Hochster and C. Huneke, Phantom homology, Memoirs of the Amer. Math. Soc., Providence, R. I., 1993.

[PS] C. Peskine and L. Szpiro, Dimension projective finie et cohomologie locale, I.H.E.S. Publ. Math., 42 (1973), 323-395.

[S] K. E. Smith, Tight closure of parameter ideals, Invent. Math., 115 (1994), $41-60$.

Department of Mathematics

University of Missouri

Columbia, MO 65211

U. S. A. 